\title{
O EFEITO DA DEPOSIÇÃO SALINA NAS ESCORRÊNCIAS RODOVIÁRIAS EM ZONAS COSTEIRAS
}

\section{THE EFFECT OF SALT DEPOSITION IN ROAD RUNOFF IN COASTAL ZONES}

\author{
Pedro Antunes ${ }^{a^{*}}$, Paulo Ramísio ${ }^{b}$ \\ ${ }^{a}$ Escola Superior de Tecnologia e Gestão de Viseu, Instituto Politécnico de Viseu, Campus \\ Politécnico, 3505-504 Viseu, Portugal \\ ${ }^{\text {b}}$ Departamento de Engenharia Civil da Escola de Engenharia da Universidade do Minho, \\ 4710-057 Braga, Portugal
}

\section{RESUMO}

As escorrências rodoviárias são uma fonte de poluição difusa linear que podem originar impactos ambientais significativos no meio recetor. Níveis elevados de deposição salina em áreas costeiras podem ter um efeito relevante nas escorrências rodoviárias. Para investigar este fenómeno um estudo de monitorização intensivo, com a recolha de mais de 200 amostras em 3 anos, foi conduzido numa autoestrada portuguesa próximo do mar. A análise de resultados baseou-se em tratamentos estatísticos, em análise exploratória de dados e na modelação do fenómeno, tendo sido incluídas variáveis associadas à meteorologia, ao troço de estrada e à deposição salina. O estudo confirma algumas especificidades das escorrências rodoviárias produzidas em áreas costeiras, como a salinidade elevada e a sua correlação com outros parâmetros. Estas características correlacionam-se com variáveis independentes associadas às condições meteorológicas, salientando-se o período seco antecedente aos eventos de precipitação e a velocidade média do vento nesse período. A modelação do fenómeno permitiu a identificação das condições associadas à maior concentração de poluentes, contribuindo deste modo para a conceção de sistemas eficazes de controlo da poluição associada a escorrências rodoviárias em zonas costeiras.

Palavras Chave - Águas de Escorrência Rodoviárias; Áreas Costeiras; Deposição Salina; Monitorização

\begin{abstract}
Road runoff is a diffuse source of pollution that can cause significant impacts in the receiving environment. High levels of salt deposition in coastal areas can have a relevant effect in road runoff. To research this phenomenon an intensive monitoring study was done in a Portuguese highway near the sea, collecting more than 200 samples over 3 years. The results were analyzed through statistical treatments in exploratory data analysis and modeling of the phenomenon, including variables associated with meteorological and traffic parameters e salt deposition. The study confirms some specifics of road runoff produced in coastal areas, such as high salinity and its correlation with other parameters. These characteristics are correlated with independent variables associated with weather conditions, highlighting the dry period prior to precipitation events and the average wind speed in that period. The modeling of the phenomenon allowed the identification of the conditions associated with the higher concentration of pollutants, thereby contributing to the design of effective pollution control systems associated with road runoff in coastal areas.
\end{abstract}

Keywords - Road runoff; coastal areas; salt deposition; monitoring

\footnotetext{
* Autor para correspondência. Corresponding author.

E-mail: baila@estgv.ipv.pt (Prof. P Antunes)
} 


\section{INTRODUÇÃO}

As escorrências rodoviárias são uma fonte linear de poluição que podem causar impactes ambientais significativos (Luker e Montague 1994, Opher e Friedler 2010). Durante os eventos de precipitação, verifica-se um processo de lavagem da bacia de drenagem das estradas, resultando na drenagem dos poluentes acumulados na plataforma e dos materiais resultantes da degradação da infraestrutura.

Investigações em escorrências rodoviárias de duas seç̧ões de estrada, localizadas em áreas costeiras portuguesas, detetaram níveis elevados de salinidade, cloretos e matéria orgânica (Antunes e Barbosa 2005). Estas evidências foram associadas à proximidade do mar e respetivos aerossóis marinhos, já que as estradas portuguesas não costeiras não apresentam este padrão (Barbosa et al. 2006).

Para Lopes e Pio (1999) as brisas marítimas são provavelmente as condições meteorológicas com maior impacto na qualidade do ar em áreas costeiras portuguesas. Referem ainda que durante a ocorrência deste fenómeno de transporte, o sal marinho é arrastado para o interior do território nacional. A distância percorrida pelos aerossóis desde o mar depende da massa das partículas, características do vento, presença de obstáculos na sua trajetória, humidade relativa e precipitação (Fitzgerald 1991). Em áreas costeiras, sais marinhos transportados pela atmosfera são depositados e permanecem no pavimento da estrada entre eventos de precipitação. Apesar desta realidade, as investigações que mencionam salinidade em escorrências rodoviárias são principalmente as que se referem a locais onde sais de degelo são aplicados no inverno (Waller e Hart 1986, Merrikhpour e Jalali 2013). Esse é porém um fenómeno diferente, o Quadro 1 apresenta as principais diferenças.

Quadro 1. Diferenças da salinidade em escorrências rodoviárias.

\begin{tabular}{ccc}
\hline Aplicação & Origem nos Sais de degelo & Origem Marinha \\
\cline { 2 - 3 } $\begin{array}{c}\text { Período de } \\
\text { Ocorrência }\end{array}$ & Antropogénico & Natural \\
\hline Compostos & Inverno & $\begin{array}{c}\text { De acordo com a variação da geração- } \\
\text { transporte de aerossóis marinhos }\end{array}$ \\
\hline $\begin{array}{c}\text { Impactos dos sais } \\
\text { no ambiente } \\
\text { recetor }\end{array}$ & $\begin{array}{c}\text { O meio ambiente recetor não } \\
\text { está sujeito a esta salinidade, } \\
\text { podendo ocorrer assim } \\
\text { impactos significativos }\end{array}$ & $\begin{array}{c}\text { Como o pavimento, o meio ambiente } \\
\text { envolvente do troço de estrada está } \\
\text { sujeito à salinidade, verificando-se a } \\
\text { adaptação do ambiente natural }\end{array}$ \\
\hline Outros poluentes & $\begin{array}{c}\text { Resíduos nos sais de degelo e e } \\
\text { matéria orgânica }\end{array}$ & $\begin{array}{c}\text { Para além da salinidade e parâmetros } \\
\text { relacionados, possivelmente matéria } \\
\text { orgânica e mais efeitos nos metais } \\
\text { pesados, pela sua maior solubilidade e } \\
\text { mobilidade }\end{array}$ \\
\hline
\end{tabular}

Quais são as características particulares das escorrências rodoviárias em áreas costeiras? Fornecer uma resposta consistente a esta questão foi uma das tarefas do Projeto G-Terra "Diretrizes para a Gestão Integrada das Escorrências rodoviárias em Portugal" (Barbosa et al. 2011).

\section{MATERIAIS E MÉTODOS}

\subsection{Caracterização da área de estudo}

A seç̧ão da autoestrada A25 monitorizada por Antunes e Barbosa (2005), a menos de 6km do mar, foi escolhida como caso de estudo piloto. Esta secção de autoestrada atravessa a Ria de Aveiro (salinidade elevada) que comunica com o Oceano Atlântico. A reduzida elevação $(5 \mathrm{~m})$ e a ausência de barreiras significativas entre o local monitorizado e o oceano potencia a ação de vento e o efeito da deposição salina (Fitzgerald 1991, Morcillo et al. 2000). Os 
ventos dominantes são de sudeste (outono-inverno) e de noroeste (primavera-verão), com uma velocidade média de $9,2 \mathrm{~m} / \mathrm{s}$ (TMUA 2014). O Quadro 2 apresenta as principais características da secção de estrada monitorizada.

Quadro 2. Características do trecho da autoestrada A25 monitorizado.

\begin{tabular}{|c|c|}
\hline Distância do mar & $5,6 \mathrm{~km}$ \\
\hline $\begin{array}{l}\text { Área da bacia de drenagem } \\
\text { monitorizada }\end{array}$ & $287,5 \mathrm{~m}^{2}$ (totalmente impermeável) \\
\hline $\begin{array}{l}\text { Características da secção de } \\
\text { estrada }\end{array}$ & $\begin{array}{c}\text { Pavimento de betume asfáltico; barreiras de segurança pré- } \\
\text { fabricadas em betão armado ( } 80 \mathrm{~cm}) \text {, tipo New-Jersey; reduzida } \\
\text { inclinação longitudinal } \\
2.5 \% \text { declive transversal; largura } 11.5 \mathrm{~m} \text { (uma direção com } 2 \\
\text { faixas de rodagem) }\end{array}$ \\
\hline Tráfego Médio Diário Anual & $\begin{array}{c}31779 \text { veículos (2008); } 32765 \text { (2009); } 33830 \text { (2010); no verão o } \\
\text { tráfego aumenta } 40 \% \text { devido ao acesso das praias }\end{array}$ \\
\hline $\begin{array}{l}\text { Principais ocupações do } \\
\text { solo }\end{array}$ & $\begin{array}{c}\text { Lagoa; oceano; porto de pescado e industria; área de esteiros e } \\
\text { marinhas; agricultura e floresta; praias; cidade de Aveiro (60000 } \\
\text { habitantes) }\end{array}$ \\
\hline
\end{tabular}

\subsection{Estação de monitorização}

De acordo com uma metodologia comum na monitorização de escorrências rodoviárias (Ramísio e Vieira 2011), no local de estudo foi instalada uma estação de monitorização, incluindo os seguintes equipamentos, operando em sincronização: um amostrador automático (ISCO 6700), um caudalímetro (do tipo borbulhador ISCO 730 associado a um descarregador Thel-mar) e um udómetro.

Uma das inovações relativamente à monitorização de escorrências rodoviárias foi a adição de um dispositivo de vela húmida. Esta metodologia, seguindo a norma ASTM G-240 (2002), foi selecionada para medir a Taxa de Deposição Salina (TDS). Um segundo dispositivo de vela húmida foi colocado na Estação Meteorológica da Universidade de Aveiro, localizada aproximadamente a $2 \mathrm{~km}$ a este da secção de estrada da A25. Neste local foram também registados os dados meteorológicos e atmosféricos.

De fevereiro de 2008 a abril de 2011 foram realizadas três campanhas de monitorização, compreendendo 30 eventos de precipitação e 215 amostras discretas de escorrências, recolhidas em diferentes condições climáticas, de tráfego e de TDS. Em cada evento oito amostras discretas, ou menos em eventos de precipitação de curta duração, foram recolhidas em frascos de borosilicato. O programa de monitorização, com um tempo total de $2 \mathrm{~h}$, tinha intervalos de tempo iniciais de 10 minutos, que eram incrementados para 20 minutos depois da terceira amostra discreta ser recolhida. Isto permitia uma melhor caracterização da escorrência inicial para avaliar a ocorrência do fenómeno do primeiro fluxo (Gupta e Saul 1996, Furumai et al. 2002, Kayhanian et al. 2012), período onde a poluição tende a estar mais concentrada, quando comparada com o restante escoamento do evento, particularmente para Períodos Secos Antecedentes (PSA) significativos.

\subsection{Métodos analíticos}

Os procedimentos de amostragem, preparação, preservação e procedimentos de análise foram conduzidos de acordo com procedimentos standard internacionais, observando um plano de Garantia e Controlo da Qualidade estabelecido pelo Projeto G-Terra, baseado no Caltrans Stormwater Monitoring Protocol Guidance Manual (Caltrans 2003) e no APHA (1998).

\subsection{Tratamento de dados}

Para tratar a variabilidade da concentração das escorrências rodoviárias no decorrer dos eventos de precipitação, foi considerada a Concentração Média por Evento (CME), representando a concentração média de um parâmetro analítico num evento, ponderada pelo volume de escorrência. A Concentração Média do Lugar (CML) de um parâmetro analítico específico representa a média dos diversos CME nos eventos monitorizados. 
Para cada poluente foi usada estatística descritiva para analisar as características estatísticas e distributivas da qualidade das escorrências rodoviárias, incluindo a verificação da ocorrência do primeiro fluxo.

A estimativa dos parâmetros relacionados com as condições do mar foi realizada com base num modelo numérico LAM (European Shelf Model) do European Centre for Medium-range Weather Forecasts (ECMWF) num ponto a oeste da praia da Barra, a aproximadamente $8 \mathrm{~km}$ da secção de estrada monitorizada.

Dados relativos aos vários eventos de precipitação monitorizados foram recolhidos e processados, conforme sumarizado no Quadro 3.

Quadro 3. Dados recolhidos associados aos vários eventos monitorizados.

\begin{tabular}{cc}
\hline $\begin{array}{c}\text { Fatores associados à } \\
\text { amostragem }\end{array}$ & $\begin{array}{c}\text { Data e início da recolha de amostras e outros dados de } \\
\text { amostragem para interpretar os resultados }\end{array}$ \\
$\begin{array}{c}\text { Precipitação, volumes e } \\
\text { caudais no evento }\end{array}$ & $\begin{array}{c}\text { Dados armazenados no data logger (precipitação e caudal); } \\
\text { intensidade de precipitação; estimativa do coeficiente de } \\
\text { escoamento; volumes parciais entre a recolha de amostras } \\
\text { discretas; hidrogramas }\end{array}$ \\
$\begin{array}{c}\text { Outros dados relacionados } \\
\text { com o evento de precipitação } \\
\text { e o PSA }\end{array}$ & $\begin{array}{c}\text { Duração do evento; PSA; duração, intensidade e volume do } \\
\text { evento de precipitação antecedente }\end{array}$ \\
$\begin{array}{c}\text { Concentrações e cargas } \\
\text { poluentes }\end{array}$ & $\begin{array}{c}\text { Concentração nas amostras discretas; média, mediana, percentis, } \\
\text { máximos, mínimos, desvio padrão, carga e CME do poluente por } \\
\text { evento; CML; polutogramas }\end{array}$ \\
\hline $\begin{array}{c}\text { Analise da ocorrência do } \\
\text { primeiro fluxo }\end{array}$ & $\begin{array}{c}\text { Gráficos: massa relativa acumulada vs. volume relativo } \\
\text { acumulado de escorrência }\end{array}$ \\
\hline
\end{tabular}

\subsection{Análise estatística multivariada e modelação}

Para conhecer a relação entre as principais variáveis e para suportar o entendimento dos processos associados, foi realizada uma análise exploratória dos dados entre os CME e entre estes e as variáveis independentes dos seguintes grupos: condições do evento de precipitação; especificidades da secção de estrada monitorizada; transporte de massas de ar e outros processos relacionados com a TDS, incluindo a dependência destes com as características climáticas antecedentes. Foram estimados os coeficientes de correlação de Pearson e ainda aplicadas as técnicas estatísticas como a Análise de Clusters e Análise dos Componentes Principais.

Realizaram-se modelações estatísticas das concentrações dos principais poluentes, aplicando a Regressão Linear Multivariada (RLM) e considerando três grupos de variáveis independentes: os parâmetros associados à meteorologia, ao troço de estrada e à deposição salina. Posteriormente foram incluídos igualmente alguns poluentes como variáveis independentes, tendo-se conseguido aumentar os coeficientes de determinação dos modelos. Foram também realizados testes à modelação e sua validação interna, incluindo a aplicação da técnica de validação cruzada.

\section{RESULTADOS E DISCUSSÃO}

A TDS tende a diminuir exponencialmente com a distância confirmando os resultados obtidos por Hossain e Easa (2011). Na secção monitorizada a TDS foi significativa, apresentando uma média de $55 \mathrm{mgCl}^{-} / \mathrm{m}^{2}$, com uma reduzida variação ao longo do ano ( $v d$. Figura 1). A TDS apresentou 2 picos, um em janeiro/dezembro e outro em maio. Neste período iniciamse os ventos mais favoráveis e a geração de aerossóis decorrente da ondulação ainda é significativa. De facto, com base no estudo realizado, a TDS parece apresentar um correlação elevada com a direção e velocidade do vento, mas sobretudo com a ondulação, sendo assim inferior no verão, apesar da direção favorável no caso de estudo. A distância ao mar, a topografia, os obstáculos no solo e a altitude da plataforma da estrada também são relevantes. 
Os eventos monitorizados representam um largo espectro de características da precipitação e das escorrências rodoviárias. A média de precipitação dos eventos foi de 5,2mm. A média da intensidade de precipitação foi de $0,047 \mathrm{~mm} / \mathrm{min}$, com um máximo de $1,02 \mathrm{~mm} / \mathrm{min}$. Verificou-se um PSA médio de 95,6h, com um mínimo de 6,7h e um máximo de 299,3h.

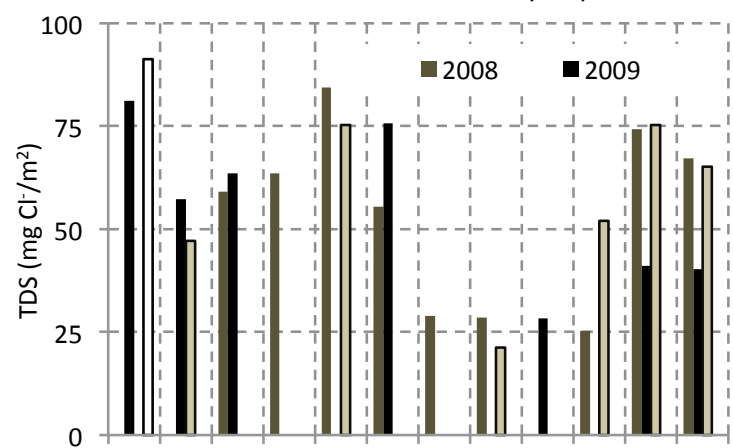

Jan Fev Mar Abr Mai Jun Jul Ago Set Out Nov Dez

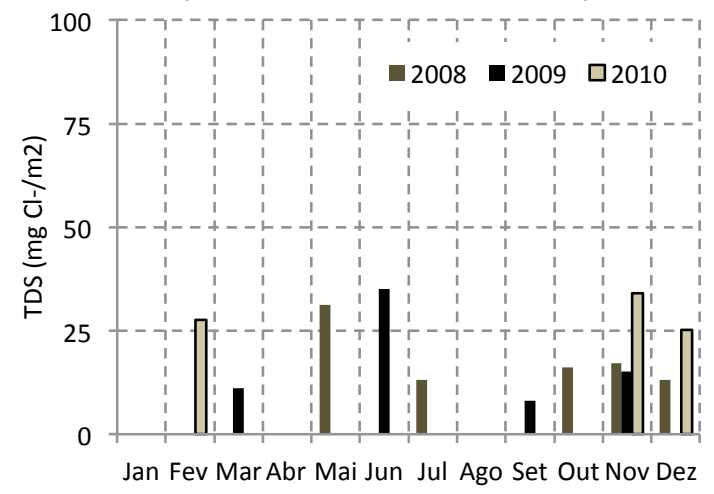

Figura 1. Variação da TDS ao longo do ano. a) estação de monitorização; b) estação meteorológica.

O Quadro 4 sumaria a estatística descritiva dos parâmetros de qualidade de água considerados relevantes para o presente estudo. O Quadro 5 apresenta a matriz dos coeficientes de correlação de Pearson entre os CME mais relevantes.

Quadro 4. Estatística descritiva das CME monitorizados.

\begin{tabular}{|c|c|c|c|c|c|c|c|c|c|c|c|c|c|}
\hline & pH & Cond & Sal & Turv & TSS & Dureza & $\mathrm{Cl}^{-}$ & CQO & COT & $\mathrm{CBO}_{5}$ & $\mathbf{F e}$ & Zn & $\mathrm{Cu}$ \\
\hline & & $(\mu \mathrm{S} / \mathrm{cm})$ & $(\mathrm{g} / \mathrm{L})$ & (FNU) & $(\mathrm{mg} / \mathrm{L})$ & $\left(\mathrm{mgCaCO}_{3} / \mathrm{L}\right)$ & $(\mathrm{mg} / \mathrm{L})$ & $\left(\mathrm{mgO}_{2} / \mathrm{L}\right)$ & $(\mathrm{mg} / \mathrm{L})$ & $\mathrm{mgO}_{2} / \mathrm{L}$ & $(\mu \mathrm{g} / \mathrm{L})$ & $(\mu \mathrm{g} / \mathrm{L})$ & $(\mu \mathrm{g} / \mathrm{L})$ \\
\hline $\begin{array}{c}\text { n.o } \\
\text { amostras }\end{array}$ & 215 & 215 & 215 & 215 & 215 & 215 & 207 & 215 & 204 & 144 & 211 & 211 & 211 \\
\hline $\begin{array}{l}\text { média } \\
\text { (CML) }\end{array}$ & 7,2 & 339,0 & 0,2 & 30,0 & 57,9 & 76,7 & 68,5 & 85,6 & 21,6 & 10,1 & 1858,0 & 139,7 & 86,9 \\
\hline med. & 7,2 & 261,7 & 0,1 & 23,6 & 44,3 & 72 & 47,3 & 74 & 14,8 & 6,4 & 1348,8 & 124,1 & 61,2 \\
\hline min. & 6,1 & 101,4 & 0,1 & 1,8 & 2,2 & 15,7 & 2,2 & 11,8 & 1,4 & 0,0 & 35,4 & 30,2 & 5,5 \\
\hline max. & 8,9 & 953,7 & 0,5 & 74,6 & 207,3 & 170,5 & 285,1 & 304,6 & 97,4 & 33,6 & 7352,2 & 406,9 & 655,8 \\
\hline DP & 0,6 & 219,6 & 0,1 & 20,0 & 48,0 & 37,6 & 70,1 & 58,5 & 20,5 & 11,1 & 1635,2 & 76,3 & 116,6 \\
\hline CV & $8 \%$ & $65 \%$ & $66 \%$ & $67 \%$ & $83 \%$ & $49 \%$ & $102 \%$ & $68 \%$ & $88 \%$ & $110 \%$ & $88 \%$ & $55 \%$ & $132 \%$ \\
\hline
\end{tabular}

Cond=condutividade; Sal=Salinidade; Turv=Turvação; $\mathrm{CQO}=$ Carência Química de Oxigénio; $\mathrm{CBO}_{5}=$ Carência Bioquímica de Oxigénio; max.= máximo; med.=mediana; $\min =$ mínimo; $\mathrm{DP}=$ desvio padrão; $\mathrm{CV}=$ coeficiente de variação

Quadro 5. Matriz dos coeficientes de Pearson e respetiva significância nos parâmetros de qualidade da água relevantes.

\begin{tabular}{|c|c|c|c|c|c|c|c|c|c|c|c|c|}
\hline & Cond & Sal & Turv & TSS & Dureza & a & CQO & COT & $\mathrm{CBO}_{5}$ & $\mathrm{Fe}$ & Zn & $\mathrm{Cu}$ \\
\hline Cond & & $0,979 * *$ & 0,140 & 0,080 & $0,392^{*}$ & $0,797^{* *}$ & $0,660 * *$ & $0,834^{* *}$ & $0,706 * *$ & 0,120 & 0,287 & $0,538 * *$ \\
\hline Sal & & & 0,145 & 0,130 & $0,405^{*}$ & $0,805^{* *}$ & $0,691 * *$ & $0,845^{* *}$ & $0,695^{* *}$ & 0,144 & 0,304 & $0,542^{* *}$ \\
\hline Turv & & & & $0,699 * *$ & 0,019 & 0,276 & 0,439* & 0,241 & 0,152 & $0,870^{* *}$ & $0,672^{* *}$ & 0,121 \\
\hline SST & & & & & $-0,130$ & 0,234 & 0,397* & 0,171 & 0,124 & $0,865^{* *}$ & $0,783^{* *}$ & 0,268 \\
\hline Dureza & & & & & & $0,397 *$ & 0,079 & $-0,000$ & $-0,290$ & $-0,060$ & $-0,110$ & $-0,160$ \\
\hline व & & & & & & & $0,430 *$ & $0,503^{* *}$ & $0,646 * *$ & 0,403* & 0,316 & 0,151 \\
\hline CQO & & & & & & & & $0,882^{* *}$ & $0,565^{*}$ & 0,342 & $0,550 * *$ & $0,783^{* *}$ \\
\hline сOT & & & & & & & & & $0,725^{* *}$ & 0,187 & $0,467 *$ & $0,837 * *$ \\
\hline $\mathrm{CBO}_{5}$ & & & & & & & & & & 0,232 & $0,480^{*}$ & $0,538^{*}$ \\
\hline $\mathrm{Fe}$ & & & & & & & & & & & $0,780 * *$ & 0,120 \\
\hline Zn & & & & & & & & & & & & $0,525^{* *}$ \\
\hline $\mathrm{Cu}_{\mathbf{u}}$ & & & & & & & & & & & & \\
\hline
\end{tabular}

A análise destes dados evidencia uma correlação forte entre a salinidade e a condutividade $(r>0.9)$. Relativamente a estes dois parâmetros os cloretos tiveram uma correlação de r 0.8. A dureza, significativa da água do mar, no presente estudo demonstrou uma correlação reduzida com a salinidade. Isto parece estar relacionado com a geologia e os solos da área envolvente. Já o pH teve valores similares à água do mar, com um coeficiente de varação muito reduzido. 
Uma análise da Matriz dos Coeficientes de Pearson evidencia uma correlação da matéria orgânica e os parâmetros associados com a salinidade. Foi relevante que as concentrações mais elevadas de CQO e COT coincidem com níveis elevados de salinidade.

O Cu, com concentrações ligeiramente mais elevadas que outras investigações sobre escorrências rodoviárias, demonstrou uma correlação relativamente significativa com a matéria orgânica e mesmo a salinidade. As concentrações de $\mathrm{Cu}$ desviam-se de outros metais pesados, como o $\mathrm{Zn}$ e o Fe. Estes últimos apresentaram as concentrações comuns detetadas em escorrências rodoviárias e a sua característica correlação elevada com os SST.

De facto, a análise estatística de dados, sendo a Análise de Clusters mais relevante para o efeito, confirmou que os parâmetros de qualidade de água podem ser divididos em dois grupos baseados nas suas correlações com (1) condutividade (geralmente na fase dissolvida da água) ou (2) SST (associados com partículas). No primeiro grupo encontra-se a Salinidade, cloretos, $\mathrm{CQO}, \mathrm{COT}, \mathrm{CBO}_{5}$, enquanto que no segundo grupo apresenta-se a Turvação, Fe, Zn.

O Quadro 6 apresenta os coeficientes de correlação de Pearson entre poluentes relevantes no estudo e variáveis independentes associadas à meteorologia e à secção da estrada.

Quadro 6. Coeficientes de Pearson correlacionando os parâmetros de qualidade e as variáveis independentes.

\begin{tabular}{|c|c|c|c|c|c|c|c|c|c|c|c|c|c|}
\hline & Cond & Salt & Turv & SST & Dureza & $\mathrm{Cl}^{-}$ & CQO & COT & $\mathrm{CBO}_{5}$ & $\mathbf{F e}$ & Zn & $\mathrm{Cu}$ & $\mathbf{P b}$ \\
\hline DE & $-0,411^{*}$ & $-0,423^{*}$ & $-0,106$ & $-0,034$ & $-0,231$ & $-0,581^{* *}$ & $-0,158$ & $-0,118$ & $-0,031$ & $-0,093$ & $-0,137$ & 0,0755 & $-0,303$ \\
\hline $\mathbf{P}$ & $-0,384 *$ & $-0,343$ & $-0,046$ & 0,221 & 0,0823 & $-0,26$ & $-0,308$ & $-0,371^{*}$ & $-0,248$ & 0,0998 & $-0,053$ & $-0,216$ & $-0,126$ \\
\hline IMP & $-0,207$ & $-0,161$ & $-0,011$ & 0,2511 & 0,1824 & $-0,053$ & $-0,238$ & $-0,347$ & $-0,229$ & 0,1312 & $-0,004$ & $-0,241$ & $-0,026$ \\
\hline IMaxP & $-0,064$ & $-0,001$ & 0,0474 & $0,619 * *$ & $-0,092$ & $-0,092$ & 0,1574 & 0,0042 & $-0,103$ & 0,3126 & 0,3593 & 0,2633 & $-0,213$ \\
\hline VE & $-0,413^{*}$ & $-0,425^{*}$ & $-0,138$ & $-0,071$ & $-0,239$ & $-0,587 *$ & $-0,181$ & $-0,140$ & $-0,023$ & $-0,123$ & $-0,163$ & 0,0702 & $-0,330$ \\
\hline PSA & $0,562 *$ & $0,552 *$ & 0,252 & 0,0975 & 0,2359 & $0,521^{*}$ & $0,425^{*}$ & $0,426 *$ & $0,606^{*}$ & 0,251 & 0,3448 & 0,2427 & 0,248 \\
\hline VPSA & $0,553 *$ & $0,541^{*}$ & 0,2481 & 0,0925 & 0,229 & $0,507 *$ & $0,414^{*}$ & $0,412 *$ & $0,597^{*}$ & 0,2448 & 0,3325 & 0,2394 & 0,2372 \\
\hline IPPSA & $-0,130$ & $-0,139$ & 0,0937 & $-0,132$ & $-0,145$ & $-0,153$ & $-0,034$ & $-0,166$ & $-0,075$ & $-0,027$ & $-0,019$ & $-0,076$ & $-0,168$ \\
\hline VVPSA & $-0,490 *$ & $-0,473^{*}$ & $-0,129$ & 0,0896 & $-0,214$ & $-0,195$ & $-0,42$ & $-0,355$ & $-0,119$ & 0,0636 & $-0,117$ & $-0,316$ & $-0,316$ \\
\hline
\end{tabular}

Os coeficientes de correlação da altura de precipitação e intensidade média de precipitação são negativos, enquanto o PSA é positivo para todos os poluentes. Isto parece indicar que o efeito de diluição induzida por precipitação intensa diminui as CME dos poluentes, incluindo a salinidade.

Como foi referido em vários estudos (e.g. Kayhanian et al. 2007, Opher et al. 2009), o PSA tem várias correlações significativas com os poluentes, sobretudo aqueles associados à matéria dissolvida e coloidal. Os veículos durante o PSA (VPSA) estão fortemente correlacionados com o PSA, e isso pode explicar o seu padrão de correlação similar.

A velocidade média do vento durante o PSA (vVPSA), parâmetro raramente considerado em estudos sobre escorrências rodoviárias, parece influenciar inversamente a salinidade, a condutividade e a matéria orgânica, demonstrando uma correlação maior do que a precipitação $(P)$ relativamente a alguns poluentes. A duração do evento (DE) correlaciona-se inversamente com os poluentes associados à salinidade.

A intensidade máxima de precipitação (IMaxP) apresenta uma correlação importante com os poluentes associados a partículas, especialmente SST. Outras variáveis - tais como a IMP e a IPPSA - não mostram uma grande correlação com os poluentes.

No presente estudo as concentrações mais elevadas de poluentes tendem a ocorrer quando, após um PSA longo, os primeiros momentos do evento são de intensidade elevada de precipitação, inflacionando o fenómeno do primeiro fluxo. Porém, a partir de um determinado PSA não ocorre um aumento muito mais significativo na poluição. 


\section{CONCLUSÕES}

Baseado num caso de estudo, foram investigadas as características e os padrões específicos das escorrências rodoviárias em áreas costeiras. Ao longo de 3 anos, um programa de monitorização intensivo foi implementado numa seç̧ão de estrada próxima do mar. Foram realizadas medidas de vários parâmetros relacionados com a meteorologia, hidrologia das escorrências rodoviárias e deposição salina (o que é inovador neste tipo de estudos).

A TDS parece seguir um padrão ao longo do ano, num compromisso entre a agitação marítima e a velocidade e a direção do vento.

Confirmou-se uma salinidade - e parâmetros associados - muito significativa nestas escorrências rodoviárias; correlacionando-se esta com a matéria orgânica, contrariamente à dureza.

As técnicas exploratórias de dados - umas mais efetivas que outras - permitiram revelar correlações entre principais os poluentes e entre estes e variáveis independentes.

A maior concentração de poluentes parece ocorrer após um longo PSA, com uma velocidade do vento reduzida e com uma intensidade de precipitação elevada ao início do evento.

\section{ESTE FACTO, CONJUNTAMENTE COM OS VALORES CARACTERÍSTICOS OBTIDOS PARA CADA PARÂMETRO DE QUALIDADE DE ÁGUA ANALISADO, E AS RESPETIVOS CME, APRESENTAM-SE COMO UM CONTRIBUTO PARA A CONCEÇÃO DE SISTEMAS DE CONTROLO DA POLUIÇÃO ASSOCIADA A ESCORRÊNCIAS RODOVIÁRIAS EM ZONAS COSTEIRAS.REFERÊNCIAS}

Antunes P., \& Barbosa, A. (2005). Highway Runoff Characteristics in Coastal Areas - A Case Study in Aveiro, Portugal. Comunicação apresentada na 10th International Conference on Urban Drainage, Copenhaga, Dinamarca.

APHA - American Public Health Association (1998). Standard Methods for the Examination of Water and Wastewater (20th ed.). Washington, D.C.: American Water Works Association/American Public Works Association/Water Environment Federation.

ASTM - American Society for Testing and Materials (2002). Standard test method for determining atmospheric chloride deposition rate by wet candle method. ASTM G140-02. ASTM International, West Conshohocken, PA, EUA.

Barbosa, A. E., Almeida, L. V., Antunes, P. B., Baguinho, R., Fernandes, J. N., Jessica, C., Ramísio, P. J., Vieira, J., Whitehead, M., \& Telhado, A. (2011) Directrizes para a Gestão Integrada das Águas de Escorrência de Estradas em Portugal. Laboratório Nacional de Engenharia Civil. Lisbon, Portugal.

Barbosa, A. E., Henriques M. J., \& Fernandes, J. (2006). Quality of highway runoff in coastal areas: special cases?. Comunicação apresentada no 8th Highway and Urban Environment Symposium, Nicósia, Chipre.

Caltrans (2003). Caltrans Comprehensive Protocols Guidance Manual. California Department of Transportation. EUA.

Fitzgerald, J. W. (1991). Marine aerosols: a review. Atmospheric Environment. Part A. General Topics, 25(3-4), 533-45.

Furumai, H., Balmer, H., \& Boller, M. (2002). Dynamic behavior of suspended pollutants and particle size distribution in highway runoff. Water Science and Technology, 46(11-12), 413-418.

Gupta, K., \& Saul, A. J. (1996). Specific relationships for the first flush load in combined sewer flows. Water Research, 30(5), 1244-1252.

Hossain, K. M. A., \& Easa, S. M. (2011). Spatial distribution of marine salts in coastal region using wet candle sensors. International Journal of Research and Reviews in Applied Sciences, 7(3), 228232.

Kayhanian, M., Fruchtman, B. D., Gulliver, J. S., Montanaro, C., Ranieri, E., \& Wuertz, S. (2012). Review of highway runoff characteristics: Comparative analysis and universal implications. Water Research, 46(20), 6609-6624. 
Kayhanian, M., Suverkropp, C., Ruby, A., \& Tsay, K. (2007). Characterization and prediction of highway runoff constituent event mean concentration. Journal of Environmental Management, 85(2), 279295.

Lopes, D. A., \& Pio, C. A. (1999). Mecanismo de interacção entre o hno3 e os aerossóis marinhos, durante os períodos de brisa. Comunicação apresentada na 6. a Conferência Nacional sobre a Qualidade do Ambiente, Lisboa, Portugal.

Luker, M., \& Montague, K. (1994). Control of pollution from highway drainage discharges. CIRIA Report. London: Construction Industry Research \& Information Association.

Merrikhpour, H., \& Jalali, M. (2013). The effects of road salt application on the accumulation and speciation of cations and anions in an urban environment. Water and Environment Journal, 27(4), 524-534.

Morcillo, M., Chico, B., Mariaca, L., \& Otero, E. (2000). Salinity in marine atmospheric corrosion: its dependence on the wind regime existing in the site. Corrosion Science, 42(1), 91-104.

Opher, T., \& Friedler, E. (2010). Fators affecting highway runoff quality. Urban Water Journal, 7(3), 155172.

Opher, T., Ostfeld, A., \& Friedler, E. (2009). Modeling highway runoff pollutant levels using a data driven model. Water Science and Technology, 60(1), 19-28.

Ramísio, P. J., \& Vieira, J. M. P. (2011). Characterization of road runoff: $A$ case study on the A3 Portuguese Highway. In S. Rauch \& G. M. Morrison \& (Eds.), Urban Environment - Proceedings of the 10th Urban Environment Symposium (pp. 285-295). Dordrecht: Springer.

TMUA - Torre Meteorológica da Universidade de Aveiro. (2014). O Clima de Aveiro. Disponível em: http://torre.fis.ua.pt/Aveiroclimate.asp, acedido a 7 de maio de 2014.

Waller, D. H., \& Hart, W. C. (1986). Solids, nutrients, and chlorides in urban runoff. In H.C. Torno, J. Marsalek \& M. Desbordes (Eds.), Urban Runoff Pollution (pp. 59-85). Berlin: Springer. 